\title{
Conocimiento y factores asociados al consumo de frutas por estudiantes universitarios de la ciudad de Medellín, Colombia
}

\author{
Knowledge and factors associated with fruit \\ consumption by university students of \\ the city of Medellin, Colombia
}

\begin{abstract}
This study aimed to describe habits, preferences, frequency of consumption and knowledge regarding fruit intake among university students in Medellin, Colombia. The sample consisted of 420 randomly chosen male and female students attending both public and private schools with ages ranging from 18-24 years. The sample was selected using a doubly stratified random sampling, with 95\% confidence level and 4\% maximum permissible error. A multivariate analysis of variance (MANOVA) with canonical contrast of orthogonal type was applied. A statistically significant difference between genders $(P<0.05)$ regarding the opinion on preference and frequency of fruit consumption was found. No difference between socioeconomic level or between attending public and private school ( $p>0.05)$ was found. The main conclusion is that university students from Medellin, Colombia, like to eat fruit.
\end{abstract}

Key words: fruit; university students; diseases; consumption; preferences.
Luis Fernando Restrepo B. (1) Luz Amparo Urango M. (2) Gloria Cecilia Deossa R. (3)

(1) Universidad de Antioquia, Facultad de Ciencias Agrarias, Escuela de Producción Agropecuaria, Grupo Investigación en Ciencias Agrarias GRICA. (2) Universidad de Antioquia, Escuela de Nutrición y Dietética, Grupo Impacto de los Componentes de los Alimentos. (3) Universidad de Antioquia, Escuela de Nutrición y Dietética. Antioquía, Colombia.

Dirigir la correspondencia a: Profesor

Luis Fernando Restrepo Betancur Carrera 75 \# 65-87 Bloque 46-102, Antioquía, Colombia

E-Mail: frbstatistical@yahoo.es

Este trabajo fue recibido el 7 de Abril de 2014 y aceptado para ser publicado el 11 de Agosto de 2014.

\section{INTRODUCCIÓN}

La importancia del consumo de frutas, es vital debido a sus propiedades nutritivas, adicionalmente por su función en la prevención de enfermedades. $(1,2)$ y han sido consumidas desde el principio de los tiempos por el hombre (3). Según la OMS la ingesta recomendada de $400 \mathrm{~g}$ per cápita diarios de frutas y hortalizas, incluidas dentro de los hábitos alimentarios, se relaciona con la reducción del riesgo de padecer enfermedades crónicas no trasmisibles (ECNT) $(2,4-7)$. La OMS, ha propuesto una estrategia orientada a la prevención y control de las mismas, ya que la ingesta insuficiente es la causante de aproximadamente 2,7 millones de muertes cada año y contribuye al aumento de la mortalidad (8-10). La Encuesta Nacional de Salud ENSIN 2010, detectó que 22\% de los colombianos consume frutas naturales de manera directa o en preparación de jugos 1 vez al día, 18,7\% los consume dos veces al día y $9,8 \%$ tres o más veces al día (11). La frecuencia en la ingesta de frutas, es importante por el aporte de nutrientes que poseen y se reemplaza el consumo de otros alimentos que pueden ser perjudiciales como es el caso de los alimentos ricos en grasas saturadas y azúcares (12-14). Es importante realizar investigaciones en diferentes grupos poblacionales, con respecto a los alimentos.
El objetivo de este estudio fué describir hábitos, gustos y frecuencia de consumo de frutas en estudiantes universitarios.

\section{SUJETOS Y MÉTODOS}

Se realizó un estudio descriptivo de tipo transversal. Donde se aplicó una encuesta la cual se sometió a juicio de expertos, sobre el consumo de frutas de acuerdo al sexo (masculino o femenino), el estrato socioeconómico (bajo, medio y alto), los gustos y conocimientos, las preparaciones y frecuencia (diaria, semanal, otra), con el fin de verificar la pertinencia de las preguntas y la metodología propuesta. El tamaño de la muestra se eligió en forma aleatoria, condicionada de acuerdo con el criterio de estratificación, utilizando una distribución uniforme, la cual fue de 420 estudiantes pertenecientes 210 de ellos a universidades públicas y 210 a privadas de la ciudad de Medellín Colombia; con participación voluntaria. Las características de los participantes en la muestra $(n=420)$ fueron estudiantes, cuyas edades oscilaron entre 18 a 24 años, hombres $(n=210)$, mujeres $(n=210)$. Se utilizó un nivel de confiabilidad del $95 \%$ y un error máximo permisible del 4,8\%, donde los parámetros estimados P y Q adoptaron el valor del $50 \%$, debido a que no se poseen estudios anteriores sobre el consumo de este grupo de alimentos en este 
tipo de población.

La base de datos se elaboró en una hoja electrónica de acuerdo con el formato delineado en la encuesta y se realizó un estricto control de calidad, con preguntas sobre el gusto y frecuencia de consumo de frutas. Los encuestadores fueron entrenados para el diligenciamiento de los formatos de recolección de la información. En el Análisis Estadístico se empleó análisis multivariado de la varianza (manova), con contraste canónico ortogonal, estableciendo la dimensionalidad de la comparación multidimensional, por medio del criterio de máxima verosimilitud observando el mayor valor propio significativo. El estudio se efectuó con variables respuestas de tipo cualitativo, las cuales están asociadas a distribuciones probabilísticas de tipo binomial y multinomial. El análisis se complementó por medio de distribuciones de frecuencia de tipo univariado y bivariado mediante tablas de contingencia; utilizando el paquete estadístico SAS versión 9,0.

\section{RESULTADOS}

El consumo de frutas fue determinado por estrato encontrando que al $95 \%$ de los estudiantes pertenecientes al estrato alto les gusta consumir frutas, en el estrato medio (91,2\%), mientras en el estrato bajo se tuvo el menor porcentaje
(44,5\%); al efectuar el análisis descriptivo según el género, se encontró similitud en el porcentaje de respuesta según el sexo, referente al consumo de frutas (63,5\% masculino, $65,3 \%$ femenino) (tabla 1). Más del $80 \%$ de los estudiantes indagados prefieren consumir frutas en ensaladas, observando un comportamiento similar $(p>0,05)$ para los 3 estratos socioeconómicos. En la tabla 1, se muestra que un $32,7 \%$ de los estudiantes universitarios de estrato bajo, consideran que las frutas pueden ser reemplazadas por otros alimentos. Adicionalmente los estudiantes de estrato alto, reconocen con más frecuencia las vitaminas que poseen las frutas, presentando diferencia estadística con respecto a los demás estratos $(p<0,05)$. Estudiantes de ambos sexos consumen en promedio una fruta al día. (Tabla 1).

Al efectuar el análisis multivariado de la varianza, el cual tiene en cuenta todas las frutas indagadas, se detectó diferencia significativa en el consumo de frutas consumidas entre estratos $(p<0,05)$, siendo los estratos alto y medio, quienes poseen mayor consumo. No se detectaron diferencias en el consumo de frutas entre tipos de universidad pública y privada, ni entre sexos $(p>0,05)$. (Tabla 2).

No se observó relación entre el sexo con respecto al gusto por las frutas; tampoco se encontró correlación con

TABLA 1

Gustos y conocimientos referentes al consumo de frutas (\%).

\begin{tabular}{|c|c|c|c|c|c|}
\hline \multirow[t]{2}{*}{ GUSTOS (Le Gusta) } & \multicolumn{2}{|c|}{ Sexo } & \multicolumn{3}{|c|}{ Estrato } \\
\hline & $M$ & $\mathrm{~F}$ & Alto & Medio & Bajo \\
\hline Consumir frutas & $63,5 \mathrm{a}$ & $65,3 a$ & $95,0 \mathrm{a}$ & $91,8 \mathrm{a}$ & $44,5 b$ \\
\hline Consume fruta en & \multicolumn{2}{|c|}{$\begin{array}{c}\text { Desayuno } \\
18,1 b\end{array}$} & \multicolumn{2}{|c|}{$\begin{array}{c}\text { Almuerzo } \\
27,2 \mathrm{a}\end{array}$} & $\begin{array}{c}\text { Cena } \\
29,5 a\end{array}$ \\
\hline Distinguir las vitaminas & $16,9 a$ & $18,0 \mathrm{a}$ & $23,3 a$ & $17,3 b$ & $16,3 b$ \\
\hline Si las frutas son reemplazables & $29,4 a$ & $11,6 b$ & $8,3 b$ & $12,0 b$ & $32,7 a$ \\
\hline \multicolumn{3}{|c|}{ Cantidad de frutas que consume (promedio día) } & & 1 & \\
\hline
\end{tabular}

Letras distintas indican diferencia significativa por fruta entre géneros y entre estratos $(p<0,05)$, se empleó el modelo lineal general $(G L M)$ para efectuar los contrastes para el gusto y conocimiento referente a las frutas.

TABLA 2

Análisis multivariado de la varianza (MANOVA). Consumo de frutas.

\begin{tabular}{|c|c|c|c|}
\hline Prueba Estadística & $\begin{array}{l}\text { Género } \\
\text { Valor p }\end{array}$ & $\begin{array}{l}\text { Estrato } \\
\text { Valor p }\end{array}$ & $\begin{array}{c}\text { Tipo Universidad } \\
\text { Valor } p\end{array}$ \\
\hline Wilks' Lambda & 0,3836 & 0,0236 & 0,7856 \\
\hline Pillai's Trace & 0,3836 & 0,0236 & 0,7856 \\
\hline Hotelling-Lawley T & 0,3836 & 0,0236 & 0,7856 \\
\hline Roy's Greatest R & 0,3836 & 0,0236 & 0,7856 \\
\hline
\end{tabular}

p>0,05 indica que no existe diferencia estadística, para las variables género, estrato y tipo de universidad.

Se utilizó análisis multidimensional de la varianza con contraste canónico ortogonal. 
la percepción de la incidencia del consumo de frutas en la salud ( $p>0,05$ ). (Tabla 3). El 80,6\% de los estudiantes del sexo masculino consideran que las frutas son benéficas para la salud, mientras el 78\% de las mujeres tienen igual opinión.

Las estudiantes universitarias prefieren las siguientes frutas en orden de mayor a menor porcentaje: el mango (96\%), la manzana (95,8\%), la mandarina $(90,5 \%)$ el banano $(87 \%)$, la fresa $(86,9 \%)$, la uva $(84,5 \%)$ y la mora $(83,5 \%)$; mientras que los estudiantes pertenecientes al sexo masculino prefieren en su orden: el banano $(93,2 \%)$, el mango (91\%), la mandarina $(90,5 \%)$, la manzana $(87,5 \%)$, la fresa $(86,2 \%)$, el limón (85\%) y la uva $(82,4 \%)$. Se presentó diferencia significativa $(p<0,05)$ a favor de las mujeres en la preferencia de las siguientes frutas: manzana, guanábana, maracuyá, papaya, noni, borojó, frambuesa y granadilla; mientras que los hombres superaron a las mujeres en el gusto por el banano, uchuva, naranja, coco, feijoa y chontaduro $(p<0,05)$. (tabla 4$)$.

Las preparaciones que más consumen con las frutas son el salpicón (frutas troceadas con helado), las cremas (producto congelado a base de frutas, leche, azúcar), las ensaladas (frutas picadas con adición de queso, salsas dulces y en ocasiones helado), las tortas y los jugos. El mayor porcentaje de consumo diario de frutas estuvo representado por los jugos, seguido de los waffles y postres, mientras que rara vez se consumen en coladas y bolis (producto congelado a base de fruta, agua y azúcar). (tabla 5).

La frecuencia de consumo de frutas diariamente se relacionó más con el banano, manzana, mandarina y mango; el consumo semanal se reportó para las frutas como el aguacate, la guayaba, la mora y el maracuyá; rara vez se consume el tamarindo, la toronja, la curuba y la sandía. (tabla 6).

\section{DISCUSIÓN}

El bajo consumo de frutas se ha relacionado con el precio que condiciona el acceso, porque el nivel socioeconómico predispone a la compra de alimentos con mayor densidad energética, como lo demostró Giskes et al 2002 (14). En nuestro trabajo, se observó que a los estudiantes de estrato socioeconómico alto (95\%) les gusta consumir fruta, lo cual está relacionado con la facilidad en poder adquirir estos alimentos.

Los resultados de nuestro estudio mostraron, que la mayoría de los estudiantes consumen una porción de fruta al día, sin encontrarse diferencia entre ambos sexos ( $p>0.05$ ); estos reportes se compararon con los obtenidos en el año 2012 en estudiantes universitarios de la carrera de Medicina, en Bogotá-Colombia (15), donde un porcentaje importante de los participantes, consumía fruta entera una vez por semana. Sin embargo la ENSIN 2010, reportó que de la población colombiana encuestada, sólo el 50,5\% consumía fruta diariamente y $35,9 \%$ de manera semanal (11), situación que se encuentra por debajo de la recomendación diaria establecida por la OMS. El consumo actual estimado de frutas y verduras es muy variable en todo el mundo, oscilando entre $100 \mathrm{~g} /$ día en los países menos desarrollados y aproximadamente 450 g/día en Europa Occidental (16). Esta situación analizada desde los resultados de nuestro estudio, muestra que siendo los universitarios una población en la que se debería practicar hábitos de consumo de alimentos saludables, es poco frecuente el consumo diario de las frutas; lo anterior quizás esté relacionado con la poca accesibilidad a este grupo de alimentos, a la necesidad de consumir mayor cantidad de energía a partir de otros alimentos y a la poca efectividad en la promoción del consumo de frutas, en los sitios donde los jóvenes pasan la mayoría del tiempo.

Los datos sobre la percepción de la incidencia del consumo de frutas en la salud, no se correlacionaron con el género, sin embargo, en el estudio de Alvarado E y Luyando JR, en 2013 en México, se evidenció que el 67\% de los adolescentes participantes relacionó la frase alimentarse sanamente con comer frutas y verduras (72\%) (17). Este dato, podría servir para un próximo estudio, donde se evalúe la percepción de alimentación saludable en población universitaria.

Los estudiantes de ambos sexos prefirieron frutas como el banano (93,2\% hombres), mango (96,0\% mujeres), manzana (95,8\% mujeres), mandarina (90,5\% ambos) y limón $(85,0 \%)$; estos datos coinciden con los reportados en el perfil nacional de consumo de frutas y verduras del año 2013(18), donde se reporta que el $50 \%$ de la población tiene un consumo de banano de 66-115 g/día y el $25 \%$ de la población consume mango por encima de $66 \mathrm{~g} /$ día, convirtiéndose en las frutas de mayor inclusión por preferencia en la canasta familiar colombiana. El consumo de estas frutas en mayor cantidad con relación a las otras, se debe a la reconocida diversidad biológica del país y por las condiciones agroecológicas de muchos de los departamentos $(18,19)$ por lo cual el cultivo de las frutas, se adaptan a diferentes suelos y condiciones climáticas.

En el caso de la manzana se destaca su consumo por la disponibilidad en las importaciones y se consume como fruta entera (18); sin embargo teniendo en cuenta que nuestro país tiene una reconocida productividad y oferta de frutas tropicales durante todo el año, se evidencia con esta situación la carencia de un adecuado fomento y consumo de las frutas

\section{TABLA 3}

Cruce de variables género vs gustos y salud.

Género Le Gustan las frutas
Si No

63,5

65,3

0,566

34,7

Prueba Chi-Dos
Porcentaje (\%)
Percepción consumo/Salud $\mathrm{Si} \quad \mathrm{No}$

57,0

43,0

Femenino

55,3

44,7

0,7288

Se utilizó la prueba Chi-2 para medir la relación entre variables, p>0,05 indica no asociación estadística. 
TABLA 4

Porcentaje por género, referente al gusto de los tipos de fruta descritos.

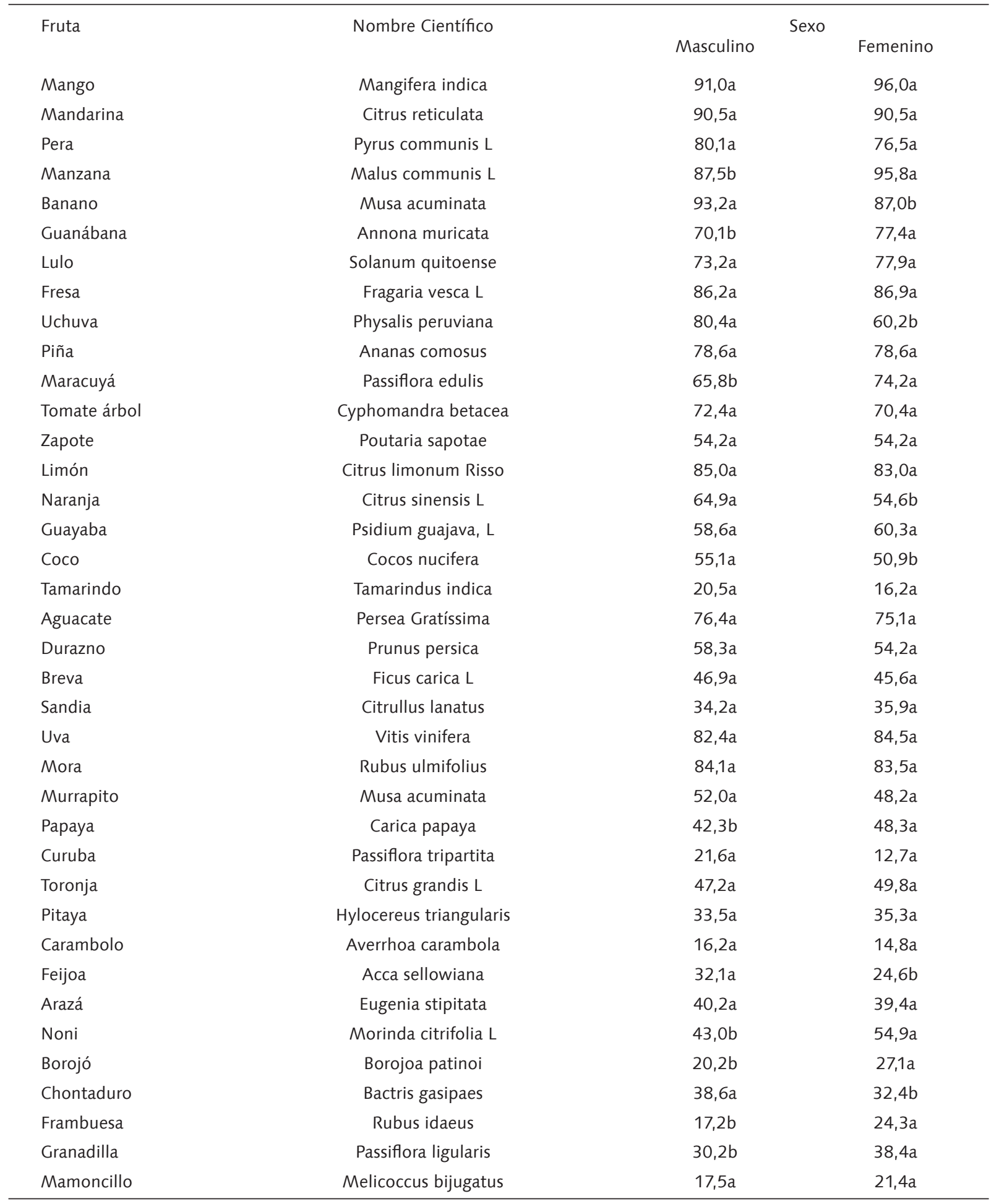

Letras minúsculas distintas indican diferencia significativa entre sexos para cada tipo de fruta $(p<0,05)$.

Se empleó el modelo lineal general (GLM) para efectuar los contrastes. 
locales, por la población colombiana. Llama la atención que siendo Colombia un país tropical, con 433 especies nativas de frutales comestibles identificados, que la hacen el primer país del mundo en biodiversidad de frutas, por kilómetro cuadrado, se encuentre un consumo tan limitado de una sola porción de frutas al día en la población estudiada; existiendo como se mencionó una oferta constante, que favorece la promoción del consumo de frutas, permitiéndole a los consumidores elegir entre la variedad para aumentar las cantidades ingeridas.

Los datos obtenidos de nuestra investigación mostraron similitud con los reportados en el año 2005 por la ENSIN, ya que entre las frutas más consumidas estaban mango (Mangifera indica), banano (Musa acuminata), guayaba (Psidium guajav, L), tomate de árbol (Cyphomandra betacea ), naranja (Citrus sinensis L), mora (Rubus ulmifolius) y limón (Citrus limonum Risso) (11); esta información confirma que la población colombiana prefiere estas frutas que son de fácil acceso por el costo.

Entre los factores determinantes socioeconómicos de los consumidores y su relación con la salud intervienen el sexo, la edad, la cultura, el entorno, las redes sociales y comunitarias. Tanto los estilos de vida como los comportamientos en salud y los factores sociales son muy importantes en la elección de los alimentos (19). El gobierno de Colombia definió a la Política Nacional de Seguridad Alimentaria y Nutricional, promulgada por el CONPES 113 (Concejo Nacional de Política Económica Social), como "la disponibilidad suficiente y estable de alimentos, el acceso y el consumo oportuno y permanente de los mismos en cantidad, calidad e inocuidad por parte de todas las personas, bajo condiciones que permitan su adecuada utilización biológica, para llevar una vida saludable y activa" (20); sin embargo a pesar de las ventajas de producción del país, los colombianos en 2005 manifestaban un bajo consumo de este tipo de alimentos; el cual se vio reflejado en la ENSIN 2005, en la cual 27,9\% y $35,3 \%$ de las personas entre los 2 y los 64 años, no incluyeron verduras ni frutas respectivamente en su alimentación diaria (21). En 2010 sólo 16,1\% de los encuestados ingerían verduras frescas diariamente y 18,3\% consumían las frutas de 3 a 4 veces por semana (11).
Para el sector de la salud, la situación de ampliar la oferta de frutas y verduras en el país, debería ser un punto a potencializar, trabajando de la mano con el sector productivo, a fin de crear sinergias que favorezcan el consumo de estos alimentos y que a su vez fortalezcan la cadena hortofrutícola del país, a través de campañas en diferentes grupos poblacionales; para dar cumplimiento a los objetivos de esta campaña mundial propuesta por la OMS (22-24).

\section{CONCLUSIONES}

Con el fin de conocer el nivel de conocimiento y la frecuencia de consumo de frutas en 420 participantes universitarios de algunas instituciones educativas de carácter público y privado de la ciudad de Medellín - Colombia, se indagó sobre el consumo de este grupo de alimentos, motivado por diversos programas como es "5 al Día" a nivel mundial. Se encontró que los participantes de este estudio del estrato alto y medio les gusta consumir frutas, caso contrario del estrato bajo. Sin embargo existe poco consumo promedio de porción de fruta al día, según el sexo y el estrato, con lo cual no logran cumplir con la recomendación sugerida por organismos internacionales de lograr un consumo de 5 al día entre frutas y verduras; no se halló diferencia significativa entre sexos.

\section{RESUMEN}

El objetivo de este estudio fue describir los hábitos, gustos, frecuencia de consumo y conocimientos relacionados con el consumo de frutas por estudiantes universitarios de Medellín, Colombia, para lo cual se aplicó una encuesta a 420 estudiantes de ambos sexos de instituciones públicas y privadas, los cuales fueron seleccionados aleatoriamente. La edad de los participantes osciló entre 18 y 24 años. Se aplicó un análisis multivariado de la varianza (MANOVA) con contraste canónico de tipo ortogonal. La muestra fue elegida por medio de la técnica denominada: muestreo aleatorio de proporciones doblemente estratificado, con base en un nivel de confiabilidad del $95 \%$ y un error máximo permisible del $4,0 \%$. No se encontró una diferencia estadística significativa por géneros en la opinión referente al gusto y frecuencia de consumo de frutas. Se detectó diferencia entre estratos y entre las universidades públicas o privadas $(p>0,05)$. La

\section{TABLA 5}

Preparaciones de frutas y frecuencia de consumo.

\begin{tabular}{|c|c|c|c|c|}
\hline Tipo & Si Consume frutas & Consumo diario & Consumo semanal & Consume rara vez \\
\hline Postres & 40,5 & 35,6 & 45,3 & 19,1 \\
\hline Jugos & 54,1 & 45,0 & 24,6 & 30,4 \\
\hline Ensaladas & 64,5 & 25,6 & 32,9 & 41,5 \\
\hline Tortas de Frutas & 60,9 & 21,9 & 16,2 & 61,9 \\
\hline Crema de Frutas & 74,5 & 9,0 & 25,0 & 66,0 \\
\hline Bolis de Frutas & 22,3 & 5,2 & 14,3 & 80,5 \\
\hline Wafles de Frutas & 52,9 & 2,1 & 6,8 & 91,1 \\
\hline Salpicón & 90,5 & 20,3 & 22,6 & 57,1 \\
\hline Coladas de Frutas & 10,1 & 0,5 & 2,9 & 96,6 \\
\hline
\end{tabular}

Se empleó análisis unidimensional de frecuencia para el consumo de frutas en distintas preparaciones. 
TABLA 6

Frecuencia de consumo de las frutas.

\begin{tabular}{|c|c|c|c|c|}
\hline \multirow[t]{2}{*}{ Fruta } & \multirow[t]{2}{*}{ Nombre Científico } & \multicolumn{3}{|c|}{$\%$ Frecuencia de Consumo } \\
\hline & & Diaria & Semanal & Rara Vez \\
\hline Mango & Mangifera indica & 39,4 & 49,1 & 11,5 \\
\hline Mandarina & Citrus reticulata & 40,5 & 50,9 & 8,6 \\
\hline Pera & Pyrus communis $L$ & 27,4 & 56,0 & 16,6 \\
\hline Manzana & Malus communis L & 65,2 & 29,1 & 5,7 \\
\hline Banano & Musa acuminata & 67,8 & 26,7 & 5,5 \\
\hline Guanábana & Annona muricata & 20,7 & 29,1 & 50,2 \\
\hline Lulo & Solanum quitoense & 33,0 & 48,2 & 15,2 \\
\hline Fresa & Fragaria vesca $L$ & 50,1 & 38,5 & 11,4 \\
\hline Uchuva & Physalis peruviana & 50,3 & 30,3 & 19,4 \\
\hline Piña & Ananas comosus & 16,8 & 47,4 & 35,8 \\
\hline Maracuyá & Passiflora edulis & 15,1 & 61,7 & 23,2 \\
\hline Tomate árbol & Cyphomandra betacea & 12,8 & 39,6 & 47,6 \\
\hline Zapote & Poutaria sapotae & 13,8 & 25,7 & 60,5 \\
\hline Limón & Citrus limonum Risso & 41,4 & 48,5 & 10,1 \\
\hline Guayaba & Psidium guajava, L & 8,3 & 63,4 & 28,3 \\
\hline Coco & Cocos nucifera & 10,8 & 36,6 & 52,6 \\
\hline Tamarindo & Tamarindus indica & 1,5 & 17,9 & 80,6 \\
\hline Aguacate & Persea Gratíssima & 14,1 & 65,7 & 20,2 \\
\hline Durazno & Prunus persica & 7,3 & 49,2 & 43,5 \\
\hline Breva & Ficus carica L & 10,2 & 36,1 & 53,7 \\
\hline Sandia & Citrullus lanatus & 1,7 & 32,1 & 66,2 \\
\hline Mora & Rubus ulmifolius & 21,1 & 62,0 & 16,9 \\
\hline Murrapito & Musa acuminata & 7,5 & 41,7 & 50,8 \\
\hline Papaya & Carica papaya & 6,1 & 33,2 & 60,7 \\
\hline Curuba & Passiflora tripartita & 3,5 & 14,2 & 82,3 \\
\hline Toronja & Citrus grandis L & 8,1 & 9,2 & 82,7 \\
\hline Pitaya & Hylocereus triangularis & 6,0 & 8,7 & 85,3 \\
\hline Carambolo & Averrhoa carambola & 1,0 & 6,4 & 92,6 \\
\hline Feijoa & Acca sellowiana & 2,0 & 23,5 & 74,5 \\
\hline Arazá & Eugenia stipitata & 0,5 & 7,7 & 91,8 \\
\hline Noni & Morinda citrifolia L & 0,9 & 22,4 & 76,7 \\
\hline Borojó & Borojoa patinoi & 3,1 & 20,8 & 76,1 \\
\hline Chontaduro & Bactris gasipaes & 4,5 & 6,9 & 88,6 \\
\hline Frambuesa & Rubus idaeus & 0,7 & 4,7 & 94,6 \\
\hline Granadilla & Passiflora ligularis & 6,3 & 27,5 & 66,2 \\
\hline Mamoncillo & Melicoccus bijugatus & 2,7 & 7,0 & 90,3 \\
\hline
\end{tabular}


principal conclusión del estudio es que a los estudiantes universitarios de la ciudad de Medellín- Colombia, les gusta consumir frutas.

Palabras clave: Frutas; universitarios; enfermedades; consumo; gustos.

Agradecimientos: Los autores expresan sus agradecimientos a los participantes que libremente permitieron a los entrevistadores, el diligenciamiento y entregaron sus datos de forma voluntaria, de igual manera a la Universidad de Antioquia.

\section{BIBLIOGRAFÍA}

1. Montaña C, Cortes M. Torija M. Agrobiodiversidad y salud. Madrid, 2008. Consultado en: www.revistaambienta.es

2. Informe de una Consulta Mixta de Expertos OMS/FAO. Dieta, nutrición y prevención de enfermedades crónicas, 2003.

3. Roman E, Cilleruelo M L. Alimentación del Niño y del Adolescente, Madrid, 2005.

4. Boutayeb A, Boutayeb S. The burden of non communicable diseases in developing countries, Int J Equity Health 2005; 4(1):2.

5. Holt E M, Steffen LM, Moran A, Basu S, Steinberger J, Ross J A, Hong Ch and Sinaiko AR. Fruit and vegetable consumption and its relation to markers of inflammation and oxidative stress in adolescents, J Am Diet Assoc 2009; 109(3): 414-21.

6. Rodríguez $M$. Consumo de frutas y hortalizas en mujeres entre 40 y 50 años de edad que realizan actividad física. Trabajo de grado. Universidad Abierta Interamericana, 2010, p 48.

7. Reiss R, Johnston J, Tucker K, DeSesso JM, Keen C. Estimation of cancer risks and benefits associated with a potential increased consumption of fruits and vegetables, Food Chem Toxicol. 2012; 50:4421-7.

8. WHO. Fruit And Vegetables Promotion Iniciative report of the meeting (Geneva) 2003

9. WHO/FAO. Fruit and vegetables for health. Report of a Joint WHO/ FAO, Japón, 2004.

10. WHO/FAO. Diet, nutrition and the prevention of chronic diseases. WHO/FAO, Geneva, 2003.

11. Fonseca $Z$, Heredia $A$, Ocampo P, Forero $Y$, Sarmiento $O$, Álvarez M, et al. Encuesta nacional de la situación nutri- cional en Colombia 2010, Primera Ed, ICBF, PROFAMILIA, MSPS, INS, p. 513, 2011.

12. Pérez $C$, Aranceta J. Consumo de Frutas y Verduras e Impacto en la Salud. Barcelona, 2006.

13. Epstein L, Gordy C, Raynor H, Beddome M, Kilanowski C, Paluch R. Increasing Fruit and Vegetable Intake and Decreasing Fat and Sugar Intake in Families at Risk for Childhood Obesity. Obes Res. 2001; 9: 171-8.

14. Giskes K, Turrell G, Patterson C and Newman B. Socioeconomic differences among Australian adults in consumption of fruit and vegetables and intakes of vitamins $A, C$ and folate. J Human Nutr Dietet. 2002 15: 375-85.

15. Becerra F, Pinzón G, Vargas M. Estado nutricional y consumo de alimentos de estudiantes Universitarios admitidos a la carrera de medicina, Rev Fac Med. 2012 ; 60 (1)

16. OMS. Estrategia mundial sobre régimen alimentario, actividad física y salud fomento del consumo mundial de frutas y verduras. Comunicado de prensa FAO. Disponible en: www.fao.org.co/cisneros.pdf.

17. Alvarado E, Luyanfo J.R. Alimentos saludables: la percepción de los jóvenes adolescentes en Monterrey, Nuevo León, Redalyc.org 2012; vol. XXI (41) 144-164

18. Perfil nacional de consumo de frutas y verduras. Minsalud y Protección Social, FAO, Bogotá, 2013.

19. Montaño P. Frutas tropicales de Colombia para el mundo: producción, agroindustria, comercialización y cadena productiva. CORPOICA, Cali, 2007.

20. Barbero L, Estudio sobre hábitos de consumo de frutas y verduras de los consumidores cordobeses. Documento de trabajo. 2012

21. Consejo Nacional de Política Económica y Social, Departamento Nacional de Planeación, Conpes 3514: política nacional fitosanitaria $y$ de inocuidad para las cadenas de frutas y de otros vegetales, Bogotá; p. 1-45. 2008.

22. ICBF, PROFAMILIA, INS, Universidad de Antioquia, Organización Panamericana de la Salud, Encuesta nacional de la situación nutricional en Colombia, ENSIN 2005, Bogotá, p. 466, 2006.

23. Winogrand, $M$, Intervenciones dentro del programa «5 al día» para promover el consumo de verduras y frutas en Argentina, Rev Chil Nutr. 2006; 33(1)

24. Marchioni D. 4to Congreso Panamericano de Promoción del consumo de verduras y frutas, Actas del Congreso 2008; 19. 